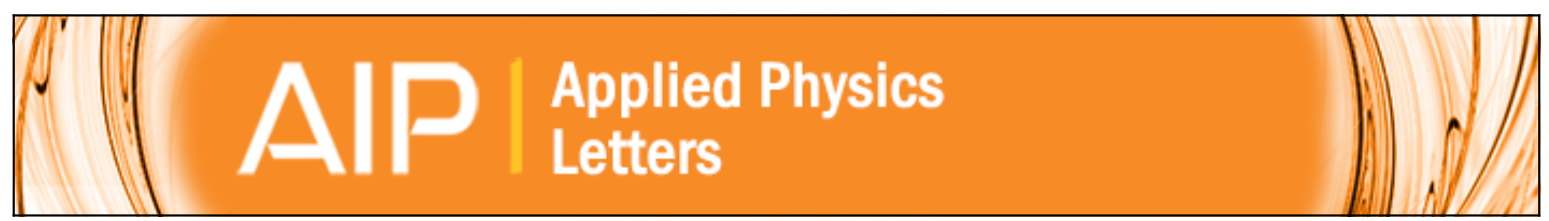

Improved photovoltaic performance of bilayer heterojunction photovoltaic cells by triplet materials and tetrapod-shaped colloidal nanocrystals doping

Yanqin Li, Rosanna Mastria, Kechang Li, Angela Fiore, Yue Wang, Roberto Cingolani, Liberato Manna, and Giuseppe Gigli

Citation: Applied Physics Letters 95, 043101 (2009); doi: 10.1063/1.3186074

View online: $\mathrm{http}: / / \mathrm{dx}$. doi.org/10.1063/1.3186074

View Table of Contents: http://scitation.aip.org/content/aip/journal/apl/95/4?ver=pdfcov

Published by the AIP Publishing

Articles you may be interested in

Identification of different origins for s-shaped current voltage characteristics in planar heterojunction organic solar cells

J. Appl. Phys. 111, 054509 (2012); 10.1063/1.3692050

Improving photovoltaic properties by incorporating both single walled carbon nanotubes and functionalized multiwalled carbon nanotubes

Appl. Phys. Lett. 94, 093509 (2009); 10.1063/1.3083544

Toward organic thick film solar cells: Three dimensional bulk heterojunction organic thick film solar cell using fullerene single crystal nanorods

Appl. Phys. Lett. 91, 173503 (2007); 10.1063/1.2801624

Enhanced photovoltaic response of organic solar cell by singlet-to-triplet exciton conversion

Appl. Phys. Lett. 90, 133509 (2007); 10.1063/1.2716209

Exciton transitions in tetrapod-shaped CdTe nanocrystals investigated by photomodulated transmittance spectroscopy

Appl. Phys. Lett. 89, 094104 (2006); 10.1063/1.2335801

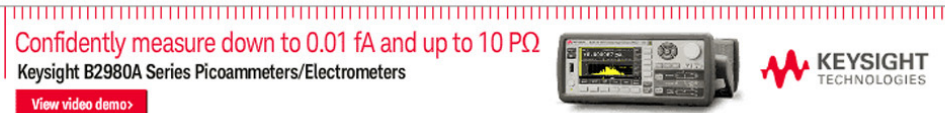




\title{
Improved photovoltaic performance of bilayer heterojunction photovoltaic cells by triplet materials and tetrapod-shaped colloidal nanocrystals doping
}

\author{
Yanqin Li, ${ }^{1, a)}$ Rosanna Mastria, ${ }^{1}$ Kechang $\mathrm{Li}^{2}{ }^{2}$ Angela Fiore, ${ }^{1}$ Yue Wang, ${ }^{2, \mathrm{~b})}$ \\ Roberto Cingolani, ${ }^{1}$ Liberato Manna, ${ }^{1}$ and Giuseppe Gigli ${ }^{1, b), c)}$ \\ ${ }^{1}$ National Nanotechnology Lab of CNR-INFM (NNL), University of Salento, Via Arnesano, \\ 73100 Lecce, Italy \\ ${ }^{2}$ State Key Lab of Supramolecular Structure and Materials, College of Chemistry, Jilin University, \\ 2699 Qianjin Avenue, Changchun 130012, People's Republic of China
}

(Received 27 May 2009; accepted 2 July 2009; published online 27 July 2009)

\begin{abstract}
The aim of this work is to investigate the photovoltaic properties of indium tin oxide/ poly (3,4-ethylenedioxythiophene):poly (styrenesulfonate) / poly(3-hexylthiophene-2,5-diyl) / fullerene/aluminum bilayer heterojunction solar cells when the active polymer layer is doped with triplet organic molecules (a platinum porphyrin complex) and tetrapod-shaped colloidal CdTe nanocrystals. In both cases, the device photovoltaic responses are greatly improved due to the enhanced triplet exciton population, in the case of molecular doping, and due to the improved charge transport and charge separation characteristics, for nanocrystal doping. The latter are related both to the relatively large nanostructured interface and to the high intrinsic carrier mobilities of nanocrystals. (C) 2009 American Institute of Physics. [DOI: 10.1063/1.3186074]
\end{abstract}

The use of conjugated polymers and fullerene as active materials in solar cells is a research area of increasing interest. $^{1-3}$ This interest is motivated by the solution processability of polymers and by the high electron mobility of fullerene. The external quantum efficiency (EQE) of such organic cells is given by $\mathrm{EQE}=\mathrm{y}_{\mathrm{A}} \quad \mathrm{y}_{\text {diff }} \mathrm{y}_{\text {diss }} \mathrm{y}_{\text {tr }} \mathrm{y}_{\mathrm{cc}},{ }^{4}$, where $\eta_{\mathrm{A}}$ is the light absorption, $\eta_{\text {diff }}$ the exciton diffusion, $\eta_{\text {diss }}$ the exciton dissociation, $\eta_{\mathrm{tr}}$ the charge carrier transport, and $\mathrm{\eta}_{\mathrm{cc}}$ the charge collection rate. Owing to the short exciton diffusion length of organic materials, exciton dissociation takes place only for excitons generated within a few nanometers from the donor-acceptor (D-A) interface. Thus, efficient photovoltaic (PV) cells have to include materials with relatively high mobility and large D-A heterojunction (HJ) interfaces for efficient exciton dissociation. ${ }^{5-7}$ Moreover, the active layer has to be thick enough to ensure strong light absorption, but sufficiently thin to allow excitons, which have very short diffusion length in organic materials, to reach the junction. In order to improve the exciton diffusion length, both high-mobility compounds and triplet materials with long exciton lifetimes can be introduced. ${ }^{8}$ An efficient organic PV HJ cell based on a triplet material, namely, 2,3,7,8,12,13,17,18-octaethyl-21H,23Hporphineplatinum(II), was demonstrated by Shao and Yang. ${ }^{9}$ Köhler et al. ${ }^{10}$ and Guo et al. ${ }^{11}$ reported platinum-polymer based triplet solar cells, while Schulz and Holdcroft ${ }^{12}$ and Yang et al. ${ }^{13}$ showed enhanced PV response due to singletto-triplet exciton conversion in conjugated polymers/iridium complexes based cells.

In this context, colloidal inorganic nanocrystals could offer processing, scale, and cost advantages of organics,

\footnotetext{
${ }^{a}$ Present address: Department of chemistry, Dalian University of Tecnology, Dalian 116024, China. Electronic mail: yanqinmm@yahoo.com.

${ }^{b)}$ Authors to whom correspondence should be addressed. Electronic addresses: yuewang@jlu.edu.cn and giuseppe.gigli@unile.it.

${ }^{c)}$ FAX: 0039-0832-298238.
}

while retaining the broadband absorption and superior transport properties of traditional PV semiconductors. ${ }^{14,15}$ For these reasons, semiconductor nanocrystals are promising building blocks for future-generation PV devices. We recently reported improved PV performance of heterostructured tetrapod-shaped (TP) $\mathrm{CdSe} / \mathrm{CdTe}$ nanocrystals using a fullerene $\left(\mathrm{C}_{60}\right)$ interlayer. ${ }^{16}$ Porter et al. ${ }^{17}$ measured the conductance of close-packed films of CdTe nanocrystals and found that the majority carriers were holes.

In this work, we investigate the effect of doping a triplet platinum dendrimer complex and CdTe TPs into the polymer active bilayer of HJ cells: (a) indium tin oxide (ITO)/ poly (3,4-ethylenedioxythiophene):poly (styrenesulfonate) (PEDOT:PSS) / poly(3-hexylthiophene-2,5-diyl) (P3HT) $\mathrm{C}_{60}$ /aluminum ( $\mathrm{Al}$ ). $\mathrm{PV}$ properties, such as excitons diffusion lengths, charge separation and charge transport have been considered. In particular, TP doping acts on exciton mobility by virtue of the superior transport properties of inorganic colloidal nanocrystals, whereas the triplet platinum dendrimer complex allows for increased triplet exciton population. We fabricated and tested ITO/ PEDOT:PSS/P3HT:CdTe/ $\mathrm{C}_{60} / \mathrm{Al}$ (b) and ITO/PEDOT:PSS/ P3HT:Pt dendrimer/ $\mathrm{C}_{60} / \mathrm{Al}$ (c) cells, in which the charge generating structure consists of a film of P3HT blended with TP or triplet materials and a thermally evaporated fullerene layer. These cells exhibited a remarkable improvement of the PV response.

P3HT (regioregular) and $\mathrm{C}_{60}(99.9 \%)$ were purchased from Sigma-Aldrich and used as received for device fabrication without further purification. The CdTe TPs were synthesized using the seeded growth approach ${ }^{18}$ and platinum dendrimers were synthesized by a published procedure. ${ }^{19}$ Five types of PV devices were fabricated: (a) ITO/PEDOT:PSS/P3HT/C $60 / \mathrm{Al}, \quad$ (b) ITO/PEDOT:PSS/ P3HT:CdTe/C $60 / \mathrm{Al}$, (c) ITO/PEDOT:PSS/P3HT:Pt dendrimer/ $\mathrm{C}_{60} / \mathrm{Al}$, (d) ITO/PEDOT: $\mathrm{PSS} / \mathrm{CdTe} / \mathrm{C}_{60} / \mathrm{Al}$, and (e) ITO/PEDOT:PSS/Pt dendrimer/ $\mathrm{C}_{60} / \mathrm{Al}$. The different ac- 

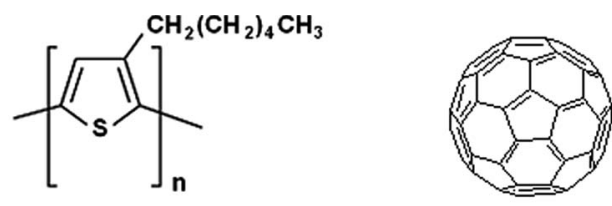

P3HT

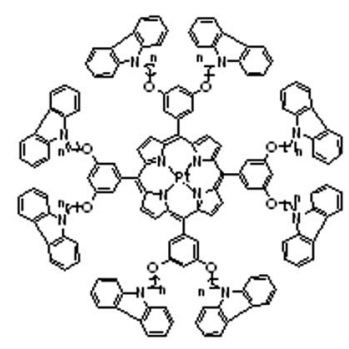

$\mathbf{c}_{60}$

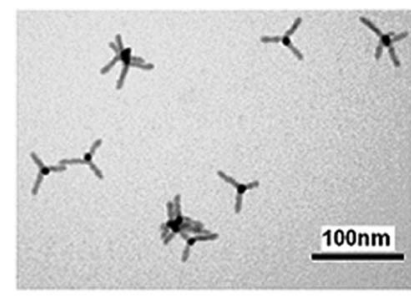

n= 4: Pt-8C4-TPP (Pt dendrimer)

TEM image of CdTe tetrapods

FIG. 1. Chemical structure of materials and TEM image of TP shaped CdTe nanocrystals.

tive layer solutions were spin cast at $1500 \mathrm{rpm}$ onto ITO glass substrates coated with $30 \mathrm{~nm}$ PEDOT-PSS, and annealed on a hot plate at $150{ }^{\circ} \mathrm{C}$ for $20 \mathrm{~min}$. Then, a $30 \mathrm{~nm}$ thick layer of $\mathrm{C}_{60}$, acting as electron acceptor and electron transport layer, was deposited by thermal evaporation at a pressure of $4.0 \times 10^{-6} \operatorname{mbar}(1 \mathrm{mbar}=100 \mathrm{~Pa})$. Finally, the samples were held at approximately $10^{-6}$ mbar overnight, after which aluminum top electrodes were deposited by thermal evaporation through a shadow mask, yielding eight individual devices with $0.08 \mathrm{~cm}^{2}$ nominal area. Absorption (Abs) measurements were carried out by using a Cary 5000 UV-visible spectrophotometer. Simulated AM1.5G illumination was realized with a Spectra Physics Oriel 150 W Solar Simulator with AM1.5G filter set. The integrated intensity was set to $100 \mathrm{~mW} / \mathrm{cm}^{2}$ using a thermopile radiant power meter (Spectra Physics Oriel, model 70260) with fused silica window. Current-voltage characteristics were carried out using the solar simulator, and a Keithley 2400 Source Measure Unit.

The chemical structures of $\mathrm{P} 3 \mathrm{HT}, \mathrm{C}_{60}$, and the synthesized platinum porphyrin dendrimer $\left(\mathrm{Pt}-8 \mathrm{C}_{4}-\mathrm{TPP}\right)$ are shown in Fig. 1. P3HT and $\mathrm{C}_{60}$ are materials with relatively high mobilities for efficient organic HJ PV cells. The platinum dendrimer complex consists of a platinum porphyrin core surrounded by a dendrimer structure of alkyl chains and carbazole side groups. In this structure, the platinum porphyrin core acts as heavy metal center, which presumably induces intersystem crossing (ISC) in the host polymer, and the external carbazole groups enhance the conductive properties. In particular, the alkyl chains provide good solubility for the dendrimers in organic solvents and excellent film forming properties by spin coating. Figure 1 reports a transmission electron microscopy (TEM) image of the CdTe TPs, synthesized with our approach, ${ }^{18}$ in which narrow distributions of the arm lengths and diameters are observed. The TP arms had average diameters of about $6.5 \mathrm{~nm}$ and average lengths of approximately $25 \mathrm{~nm}$.

Figure 2(a) shows the absorption spectra of devices with different active layers. The use of P3HT and $\mathrm{C}_{60}$ allows for excellent film forming properties, and more importantly, for high extinction coefficients in the visible region, i.e., absorption spectra covering the whole visible spectrum range from
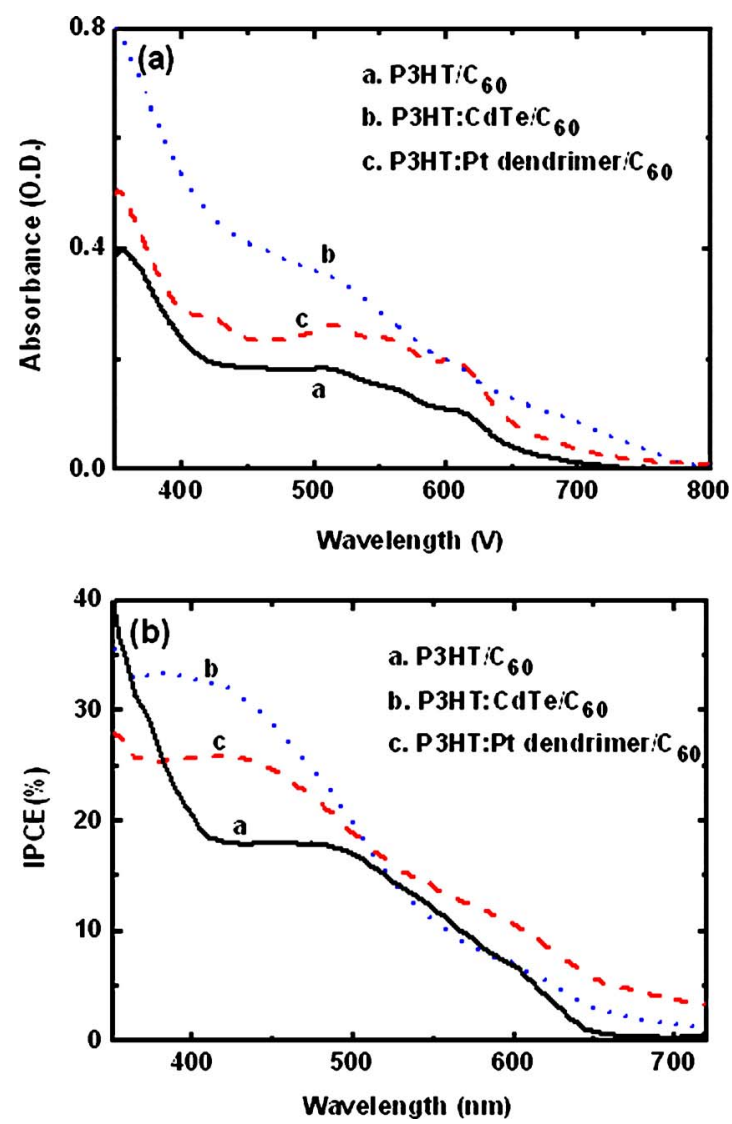

FIG. 2. (Color online) (a) Absorption spectra of devices with active layer structures: $\mathrm{P} 3 \mathrm{HT} / \mathrm{C}_{60}$ (solid), P3HT:CdTe/C 60 (dot), and P3HT:Pt dendrimer/ $\mathrm{C}_{60}$ (dash). (b) IPCE spectra of devices with different active layer structures.

380 to $750 \mathrm{~nm}$ [see Fig. 2(a)-a]. When P3HT is doped with CdTe TPs optical absorption is further extended up to 820 nm [Fig. 2(a)-b]. In more detail, an intense Soret band of $\mathrm{Pt}$ dendrimer is observed at $404 \mathrm{~nm}$ [Fig. 2(a)-c] and two $Q$ bands at 540 and $510 \mathrm{~nm}$ were overlapped with the strong absorption spectrum of P3HT. ${ }^{20}$

In order to investigate the influence on the PV properties of doping the polymer layer in the HJ bilayer cells, we fabricated two sets of devices in which the P3HT polymer layer was doped with triplet Pt dendrimers and CdTe TPs. As shown in Fig. 3(a) and Table I(a), before doping the P3HT layer, the device exhibited relatively low short circuit current density $\left(J_{\mathrm{sc}}\right)$, low open circuit voltage $\left(V_{\mathrm{oc}}\right)$, small fill factor $(\mathrm{FF})$, and low power conversion efficiency (PCE). The $J_{\mathrm{sc}}$, $V_{\mathrm{oc}}$, and FF of the device are $2.5 \mathrm{~mA} / \mathrm{cm}^{2}, 0.2 \mathrm{~V}$, and 0.32 , respectively, yielding a PCE of $0.17 \%$. An efficiency-limiting process in such devices is the short exciton diffusion length and the occurrence of the charge separation processes only in the limited interfacial region between the P3HT and $\mathrm{C}_{60}$ layers. Hence, regions of $\mathrm{P} 3 \mathrm{HT}$ and $\mathrm{C}_{60}$ layers away from the interface produce no charges but act only as a charge carrier transporting media.

$\mathrm{PV}$ devices based on $\mathrm{CdTe} / \mathrm{C}_{60}$ active layers, in which CdTe TPs and $\mathrm{C}_{60}$ layers work as electron donor and electron acceptor/transport layers, respectively, were also fabricated. As shown in Table $\mathrm{I}(\mathrm{d})$, the improved $J_{\mathrm{sc}}\left(3.1 \mathrm{~mA} / \mathrm{cm}^{2}\right), V_{\mathrm{oc}}$ $(0.33 \mathrm{~V}), \mathrm{FF}(0.32)$, and PCE $(0.33 \%)$ of the device can be attributed to the efficient charge separation and to the enhanced charge transport induced by the relatively large nanostructured interface and the high intrinsic carrier mobilities 

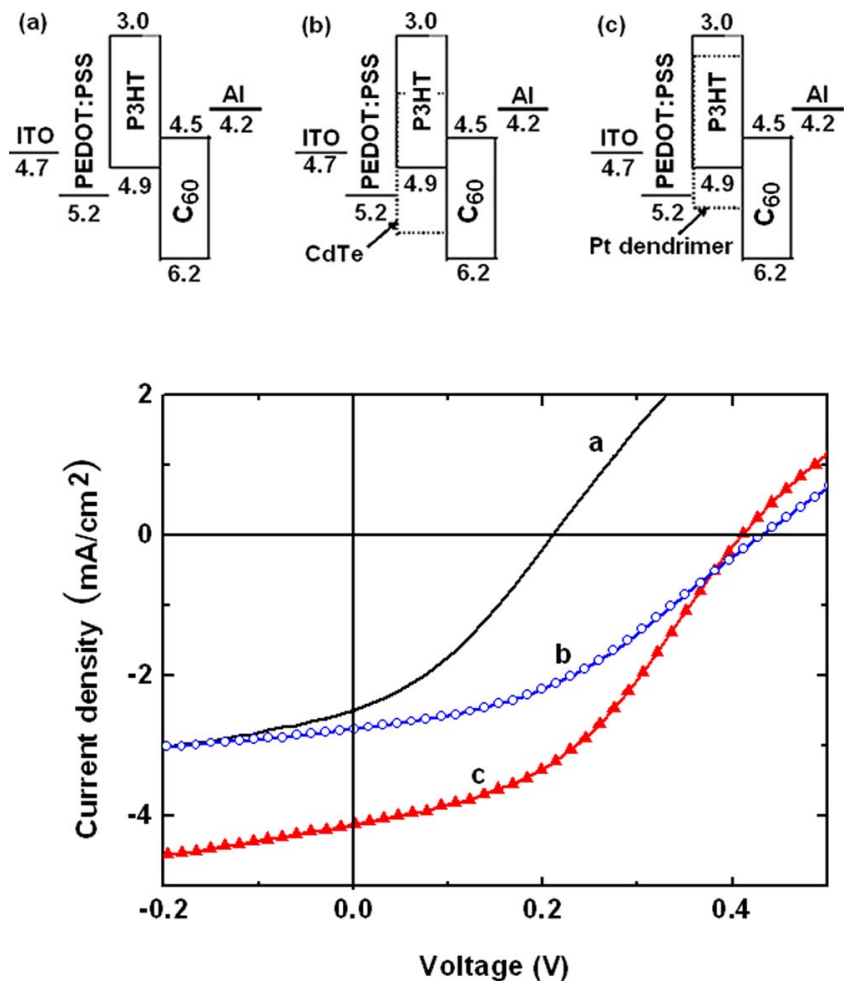

FIG. 3. (Color online) Energy band diagrams and current-voltage characteristics of devices: (a) ITO/PEDOT:PSS/P3HT/ $\mathrm{C}_{60} / \mathrm{Al}$ (solid), (b) ITO/PEDOT:PSS/P3HT:CdTe/C ${ }_{60} / \mathrm{Al}$ (circle), (c) ITO/PEDOT:PSS/ $\mathrm{P} 3 \mathrm{HT}$ :Pt dendrimer/ $\mathrm{C}_{60} / \mathrm{Al}$ (triangle).

of the nanocrystals, respectively. The effect of doping the polymer layer has been first evaluated by blending CdTe TPs with P3HT. As shown in Fig. 3(b) and in Table I(b), the PV performances of such devices were greatly improved with respect to those of $\mathrm{P} 3 \mathrm{HT} / \mathrm{C}_{60}$ and $\mathrm{CdTe} / \mathrm{C}_{60}$ based devices. The $J_{\mathrm{sc}}, V_{\mathrm{oc}}$, and FF of the device were $2.8 \mathrm{~mA} / \mathrm{cm}^{2}, 0.44 \mathrm{~V}$, and 0.38 , respectively, yielding a PCE of $0.47 \%$. Figure 2(b)-b shows the wavelength dependence of the incident photon-to-current conversion efficiency (IPCE) for the devices. Compared to the $\mathrm{CdTe} / \mathrm{C}_{60}$ based devices discussed above the efficiency is improved, probably due to the good percolation pathway to the electrodes and to the increased fraction of light harvested by the combination of polymer and nanocrystals. Compared to the $\mathrm{P} 3 \mathrm{HT} / \mathrm{C}_{60}$ based devices, we attributed the enhanced efficiency to the high carrier mobility of TPs and to the increase of charge carriers, induced by the nanostructured interface.

Finally, we fabricated triplet PV cells in which the active layer is constituted by pure $\mathrm{Pt}$ dendrimer/ $\mathrm{C}_{60}$. For this class of devices, we obtained $0.37 \mathrm{~mA} / \mathrm{cm}^{2}, 0.38 \mathrm{~V}$, and 0.32 , for $J_{\mathrm{sc}}, V_{\mathrm{oc}}$, and FF, respectively, yielding a PCE of $0.05 \%$ as shown in Table I(e). The low $J_{\mathrm{sc}}$ and PCE are attributed to

TABLE I. Summary of device characteristics measured under simulated solar illumination $\left(\mathrm{AM} 1.5 \mathrm{G}, 100 \mathrm{~mW} / \mathrm{cm}^{2}\right)$.

\begin{tabular}{|c|c|c|c|c|}
\hline Active layer structure & $\begin{array}{c}J_{\mathrm{sc}} \\
\left(\mathrm{mA} / \mathrm{cm}^{2}\right)\end{array}$ & $\begin{array}{l}V_{\mathrm{oc}} \\
(\mathrm{V})\end{array}$ & $\begin{array}{l}\mathrm{FF} \\
(\%)\end{array}$ & $\begin{array}{l}\text { PCE } \\
(\%)\end{array}$ \\
\hline (a) $\mathrm{P} 3 \mathrm{HT} / \mathrm{C}_{60}$ & 2.546 & 0.214 & 0.32 & 0.17 \\
\hline (b) P3HT:CdTe/ $\mathrm{C}_{60}$ & 2.775 & 0.442 & 0.38 & 0.47 \\
\hline (c) P3HT:Pt dendrimer $/ \mathrm{C}_{60}$ & 4.152 & 0.412 & 0.41 & 0.70 \\
\hline (d) $\mathrm{CdTe} / \mathrm{C}_{60}$ & 3.100 & 0.336 & 0.32 & 0.33 \\
\hline (e) $\mathrm{Pt}$ dendrimer/ $\mathrm{C}_{60}$ & 0.366 & 0.381 & 0.32 & 0.05 \\
\hline
\end{tabular}

the poor mobility of the Pt dendrimer film. To overcome this problem we blended the triplet platinum dendrimer complex with P3HT, obtaining a remarkable improvement of the PV response. As shown in Fig. 3(c) and Table I(c), the $J_{\mathrm{sc}}, V_{\mathrm{oc}}$, and $\mathrm{FF}$ of the Pt dendrimer/P3HT device are $4.2 \mathrm{~mA} / \mathrm{cm}^{2}$, $0.4 \mathrm{~V}$, and 0.41 , respectively, yielding a PCE of $0.70 \%$. Figure 2(b)-c shows the IPCE spectrum for the device. The platinum dendrimer compounds were selected because the heavy metal atoms determine efficient ISC processes from singlet to triplet excitons, ${ }^{11,13}$ which are characterized by longer lifetime. Thus, by properly doping high mobility polymers, such as $\mathrm{P} 3 \mathrm{HT}$, with triplet organic materials, it is possible to increase the exciton diffusion length (related to the exciton lifetime), while retaining a reasonably high charge carrier mobility. This approach allows for a remarkable improvement of the PV performances.

In conclusion, we investigated the effect induced on the PV properties of active bilayer ITO/PEDOT:PSS/ $\mathrm{P} 3 \mathrm{HT} / \mathrm{C}_{60} / \mathrm{Al} \mathrm{HJ}$ cells by doping the active polymer layer either with triplet materials or with TPs. Our results show that PV response of the device was greatly improved in both cases. In the first case the increase of PV performances is related to the increased exciton diffusion length due to the enhanced triplet exciton population. In the second case the superior efficiency arises from the efficient charge separation and the enhanced charge transport due to the relatively large nanostructured interface and high intrinsic carrier mobilities of the inorganic semiconductor nanocrystals, respectively.

${ }^{1}$ Y. Kim, M. Shin, I. Lee, H. Kim, and S. Heutz, Appl. Phys. Lett. 92, 093306 (2008).

${ }^{2}$ K. Kim, J. Liu, and D. L. Carroll, Appl. Phys. Lett. 88, 181911 (2006).

${ }^{3}$ S. W. Tong, C. F. Zhang, C. Y. Jiang, Q. D. Ling, E. T. Kang, D. S. H. Chan, and C. X. Zhu, Appl. Phys. Lett. 93, 043304 (2008).

${ }^{4}$ B. R. Saunders and M. L. Turner, Adv. Colloid Interface Sci. 138, 1 (2008).

${ }^{5}$ G. Yu, J. Gao, J. C. Hummelen, F. Wudl, and A. J. Heeger, Science 270, 1789 (1995).

${ }^{6}$ P. Peumans, S. Uchida, and S. R. Forrest, Nature (London) 425, 158 (2003).

${ }^{7}$ S. Yoo, B. Domercq, and B. Kippelen, Appl. Phys. Lett. 85, 5427 (2004).

${ }^{8}$ P. Schilinsky, C. Waldauf, and C. J. Brabec, Appl. Phys. Lett. 81, 3885 (2002).

${ }^{9}$ Y. Shao and Y. Yang, Adv. Mater. 17, 2841 (2005).

${ }^{10}$ A. Köhler H. F. Wittmann, R. H. Friend, M. S. Khan, and J. Lewis, Synth. Met. 77, 147 (1996).

${ }^{11}$ F. Q. Guo, Y. G. Kim, J. R. Reynolds, and K. S. Schanze, Chem. Commun. (Cambridge) 2006, 1887.

${ }^{12}$ G. L. Schulz and S. Holdcroft, Chem. Mater. 20, 5351 (2008).

${ }^{13}$ C. M. Yang, C. H. Wu, H. H. Liao, K. Y. Lai, H. P. Cheng, S. F. Horng, H. F. Meng, and J. T. Shy, Appl. Phys. Lett. 90, 133509 (2007).

${ }^{14}$ A. P. Alivisatos, J. Phys. Chem. 100, 13226 (1996).

${ }^{15}$ C. B. Murray, C. R. Kagan, and M. G. Bawendi, Annu. Rev. Mater. Sci. 30, 545 (2000).

${ }^{16}$ Y. Q. Li, R. Mastria, A. Fiore, C. Nobile, L. X. Yin, M. Biasiucci, G. Cheng, A. M. Cucolo, R. Cingolani, L. Manna, and G. Gigli, "Improved photovoltaic performance of heterostructured tetrapod-shaped CdSe/CdTe nanocrystals using C60 interlayer," Adv. Mater. (to be published), DOI: 10.1002/adma.200901338.

${ }^{17}$ V. J. Porter, T. Mentzel, S. Charpentier, M. A. Kastner, and M. G. Bawendi, Phys. Rev. B 73, 155303 (2006).

${ }^{18}$ A. Fiore, R. Mastria, M. G. Lupo, G. Lanzani, C. Giannini, E. Carlino, G. Morello, M. D. Giorgi, Y. Q. Li, R. Cingolani, and L. Manna, J. Am. Chem. Soc. 131, 2274 (2009).

${ }^{19}$ C. Huo, H. D. Zhang, H. Zhang, H. Y. Zhang, B. Yang, P. Zhang, and Y Wang, Inorg. Chem. 45, 4735 (2006).

${ }^{20}$ Y. Q. Li, A. Rizzo, M. Salerno, and M. Mazzeo, Appl. Phys. Lett. 89, 061125 (2006). 